neurocognitive deficit following coronary artery bypass grafting (CABG).

Neurocognitive function was assessed using cognitive P300 evoked potentials in 104 patients undergoing CABG with cardiopulmonary bypass. Trailmaking Test A (TTA) and Mini Mental State Examination (MMSE) psychometric tests were also performed and all assessments were repeated at 7 days, 4 months and 3 years. Patients matched for age and sex $(n=80)$ were used as nonsurgical controls.

Compared to preoperative values, P300 evoked potentials in the CABG group were significantly extended at each stage of followup, indicating that neurocognitive function was impaired. Notably, postoperative cognitive measures in these patients were also significantly impaired by comparison with the controls at each stage. Regression analysis revealed predictive variables for long-term neurocognitive deficit: neurocognitive deficit at 4 months' follow-up, age, and persistent atrial fibrillation. MMSE results indicated that patients had no overt neurologic disorders or dementia, whereas TTA results in CABG patients were significantly abnormal at 3 years.

Having demonstrated both objectively and by comparison with controls that $\mathrm{CABG}$ causes long-term neurocognitive deficit, the authors suggest that individual strategies should be developed to treat patients at increased risk.

Original article Zimpfer D et al. (2004) Neurocognitive deficit following coronary artery bypass grafting: a prospective study of surgical patients and nonsurgical controls. Ann Thorac Surg 78: 513-519

\section{Risks and benefits of warfarin plus aspirin}

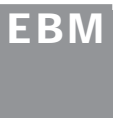

There is a lack of consensus on whether patients starting warfarin therapy should continue taking daily aspirin. Although antithrombotic protection is likely to be superior when both agents are used, some clinicians fear that the risk of increased bleeding is unacceptably high. In an attempt to resolve this problem, Larson and Fisher have carried out a systematic review and meta-analysis of nine randomized trials comparing warfarin plus aspirin versus warfarin alone.
There were five trials covering mechanical heart valves, of which four used the same INR in both treatment groups. The pooled results of these four studies indicated that, compared with warfarin alone, the combination of warfarin and aspirin significantly decreased the rates of thromboembolic events and all-cause mortality, while increasing major bleeding. The fifth mechanical-heart-valve trial, in which a reduced INR was used in the warfarin plus aspirin group, showed no difference in the rate of thromboembolic events but a decrease in major bleeding and all-cause mortality with combination therapy. Three trials covering secondary prevention of myocardial infarction showed no significant differences in subsequent myocardial infarction, major bleeding or all-cause mortality between the treatment groups. The single trial covering atrial fibrillation was terminated early and conclusions could not be drawn from the results.

In conclusion, Larson and Fisher's analysis suggests that combination therapy with warfarin plus aspirin is appropriate for mechanical heart valve patients, whereas there are insufficient data to guide this decision in other common warfarin indications.

Original article Larson RJ and Fisher ES (2004) Should aspirin be continued in patients started on warfarin? J Gen Intern Med 19: 879-886

\section{Diagnosis of infective endocarditis}

It has been suggested that transthoracic echocardiography (TTE) is overused in patients with suspected infective endocarditis. Vieira et al. have evaluated the frequency and diagnostic yield of repeated TTE, and also of transesophageal echocardiography (TOE), in a prospective cohort study.

Patients with suspected infective endocarditis $(n=262)$ were all submitted to a first TTE and TOE examination. Repeat examinations were performed when a diagnosis could not be made on the basis of previous results. The team assessed the diagnostic contribution of the examinations relative to the diagnosis of endocarditis.

The 262 patients experienced a total of 266 episodes of suspected endocarditis. TTE was performed more than once in 192 (72.2\%)

\section{GLOSSARY}

INR

International normalized ratio, which is a method used to compare the activity of different batches of thromboplastin 